\title{
Hannah Arendt lectora de Rousseau
}

\author{
Julia G. Smola \\ juliasmola@yahoo.com.ar \\ Universidade de General Sarmiento, Buenos Aires, Argentina
}

resumen Estamos acostumbrados a ver en Rousseau al primer pensador moderno de la libertad. Sin embargo, la versión de Rousseau que nos transmite Arendt, y que aquí intentaremos rastrear, dista mucho de esta imagen. Digamos que si Arendt podría acordar con la sentencia corriente de que Rousseau formuló la idea de libertad que trascendió en el lenguaje político moderno, esto no lo convierte en un pensador de la libertad política. Rousseau es quien, para Arendt, contribuye en mayor medida a la moderna teoría de la soberanía, que ella "lleva a una negación de la libertad humana". Pero en su crítica, Arendt nos exime de una lectura erudita sobre la teoría de Rousseau para mostrarnos las consecuencias que tuvo cierta apropiación de sus ideas sobre las experiencias políticas de la modernidad. A través de esta crítica podemos rastrear cómo se define su propia concepción de la política en la modernidad.

palabras claves Arendt; Rousseau; Soberanía; Voluntad; Contractualismo Libertad

"El hombre ha nacido libre y en todas partes de encuentra encadenado." (ROUSSEAU, J-J. 1993, I, 1) Rousseau abre con esta famosa sentencia sus reflexiones del Contrato Social. A partir de allí, queda claro que se trata de un autor cuya preocupación declarada es denunciar la tiranía y que, a diferencia de Thomas Hobbes se rehúsa a abrazar la servidumbre que reina por todas partes en nombre de la seguridad y el orden establecidos. En efecto, estamos acostumbrados a ver en Rousseau el primer pensador moderno de la libertad, tanto por su denuncia de las diversas formas de dominación política y social como por el papel que sus ideas jugaron en 


\section{4}

la Revolución Francesa. Sin embargo, la versión de Rousseau que nos transmite Hannah Arendt, y que aquí intentaremos rastrear, dista mucho de esta imagen. Digamos que si, en principio, Arendt podría acordar con la sentencia corriente de que Rousseau formuló la idea de libertad que trascendió en el lenguaje político moderno, este hecho no lo convierte en un pensador de la libertad política. Rousseau es quien, para Arendt, contribuye en mayor medida a la identificación moderna entre libertad y soberanía, identificación peligrosa y perniciosa para nuestra concepción de la política puesto que ella "lleva a una negación de la libertad humana -es decir, si se comprende que, sean lo que sean, los hombres jamás son soberanos-, o bien a la idea de que la libertad de un hombre, de un grupo o de una entidad política se puede lograr sólo al precio de la libertad -o sea, la soberanía- de todos los demás." (ARENDT, 1996, p. 177) Es decir que la idea de libertad-soberana conduce a la conclusión de que en la política moderna “o nunca hubo libertad o ya nunca más habrá libertad.” (TASSIN, 2007, p. 309)

Al recorrer las páginas de Sobre la revolución percibimos la importancia política de la crítica a la voluntad como concepto principal de una teoría de la soberanía. Este pensamiento confunde la política con el ejercicio de un poder absoluto, confunde a su vez dicho poder con la dominación, y a éste con la coerción y la fuerza. La idea de voluntad se encuentra en el origen de estos presupuestos haciendo de la coerción y la dominación el resultado del ejercicio legítimo del poder (ARENDT, 1996, p. 163-177; TASSIN, 2007, p. 301-315).

Pero en estas páginas también se confunde la lectura crítica de Arendt sobre las ideas de Rousseau, con la pregnancia que estas ideas tuvieron en el pensamiento revolucionario, en particular, sobre Robespierre y Sieyès. En efecto, en las páginas de Sobre la Revolución la autora analiza las ideas de Rousseau -como el concepto deVoluntad General, el de Nación, pero también el de Pueblo, y las pasiones de la piedad y compasión que lo unen a la elite revolucionaria- en el contexto de la Revolución Francesa y a través de sus protagonistas e ideólogos. Así, Arendt nos exime de una lectura erudita sobre la teoría de Rousseau para mostrarnos las consecuencias que tuvo cierta apropiación de sus ideas sobre las experiencias políticas de la modernidad. En su crítica a estas lecturas, Arendt traza un contra Rousseau (TASSIN, 2007, p. 309-313) a través del cual se 
define su propia concepción de la política. Así es que, al recorrer la lectura que Arendt hace sobre ciertas ideas de Rousseau accedemos menos a una imagen del Rousseau de Arendt que a su propia concepción política y a una teoría "en espejo" del ejercicio político de la libertad del pueblo como no-soberano. ${ }^{1}$

\section{En el nombre del pueblo: voluntad general versus voluntad de todos}

El nudo de la crítica arendtiana se plantea alrededor de la distinción que hace Rousseau entre la "voluntad de todos" y la "voluntad general". En primer lugar, el problema es que, mientras que la voluntad de todos presupone un cuerpo político constituido y dentro del cual estas diferentes "voluntades" puedan confrontarse, la voluntad general representa el acto mismo de la constitución del cuerpo político. El pueblo se constituye en la voluntad general a partir de la sumisión de las particularidades a esta última. De esta manera y lejos de ser una pluralidad que se constituye en el momento de su encuentro, el pueblo puede aparecer sólo bajo la figura de la unidad que se forma, no como una alianza (societas) de aquellos diferentes, sino a pesar de o contra estas diferencias. En segundo lugar, entonces, se encuentra el problema de la substitución de la voluntad a la "elección deliberada y la opinión reflexiva" del pueblo (ARENDT, 1992, p. 77). La voluntad "excluye, por naturaleza, todo proceso de confrontación de opiniones y el de su eventual concierto." (ARENDT, 1996, p.171) El pueblo debe, por lo tanto, no solamente ser uno sino también ser mudo.

Veamos un poco más en detalle estas dos consecuencias del pensamiento rousseauniano. La idea de voluntad general sutura la división social a través de la identificación del ser nacional con la figura del pueblo. En el análisis de Arendt, fueron las condiciones políticas, y no alguna teoría particular, las que llevaron al reemplazo de las preocupaciones por la "República" a la defensa de los intereses del "Pueblo". ${ }^{2}$ La brecha social y política entre gobernantes y gobernados, entre la república y la nación, no fue alterada por la caída de la monarquía, y la liberación de la tiranía hizo poco por liberar a los hombres del yugo de la necesidad. Este se convirtió 
en el objetivo de la revolución y allí encontraron los revolucionarios un lazo que fuera capaz de superar la brecha que los separaba del pueblo, ya que los gobiernos revolucionarios no eran "ni del pueblo ni por el pueblo, sino, en el mejor de los casos, para el pueblo.” (ARENDT, 1992, p. 75) El Pueblo entonces y no la República; pero también un pueblo unificado por sus carencias. Según Arendt, los hombres de la revolución francesa sólo debieron identificar a la nación con los padecimientos de la mayoría: le peuple, les malheureux. La razón de esta identificación es simple, y lógicamente irrebatible, sólo la necesidad puede decir algo al unísono, sólo en cuanto seres carentes y sujetos a necesidades vitales los hombres aparecen como una unidad. Pero esta unidad que parece natural no es tal por fuera del ámbito de las necesidades vitales de todos los hombres. No lo es en el ámbito político donde no existe el hombre sino los hombres (ARENDT, 2007 , p. 45). Fuera del ámbito de las necesidades vitales, la unidad de la voluntad del pueblo requiere superar la escisión que caracteriza estructuralmente a la voluntad. Frente a la formulación rousseauniana del alma escindida podemos tener la impresión de que se trata de dos voluntades distintas y por lo tanto en posible diálogo, pero en realidad -dice Arendtse trata de una voluntad escindida en dos: la voluntad particular y la voluntad general, que no dialogan entre sí sino que entran en conflicto haciendo que una sea derrotada por la otra. "[El] dos en uno de la soledad que pone en marcha el proceso del pensamiento tiene el efecto opuesto sobre la voluntad: la paraliza y la cierra dentro de sí misma; querer en soledad es siempre velle y nolle, querer y no querer al mismo tiempo."(ARENDT, 1996, p.171)

El reemplazo de la deliberación y del acuerdo externo producto de la puesta en escena del conflicto social, acuerdo expresado en la formulación rousseauniana de la voluntad de todos, por la coincidencia interna de la voluntad consigo misma no resulta de la observación de la naturaleza de los hombres sino del desconocimiento de la pluralidad humana así como de las condiciones en que la política es posible. La visión de la miseria proyectó esta imagen de unidad natural ya que aquellos que estaban siendo observados desde fuera por quienes no compartían estos padecimientos, carecían de todo y se encontraban, por así decir, despojados de todo derecho y pertenencia social o política. Pero esta unidad, denuncia Arendt, no tiene nada de natural y se logra únicamente por la 
reducción de la pluralidad a la alteridad. En efecto, tal como señala Margaret Canovan, a pesar de las aparentes afinidades, la divisoria de aguas entre Arendt y Rousseau se traza a partir del concepto de pluralidad (CANOVAN, 1983, p. 290-292). En el pensamiento de Rousseau, la pluralidad de voluntades particulares es suplantada por la idea de intereses particulares y opuestos al bien común. De esta forma, la lucha contra la miseria requiere declarar la guerra al egoísmo de la sociedad, y éste puede ser identificado como la parte interna de cada uno que le dicta sus propios intereses.

Pero la única lógica por medio de la cual dos voluntades distintas pueden unirse, es enfrentándose contra una tercera, y ésta es la base de todos los nacionalismos pasados y por venir. Rousseau -dice Arendt"Deseaba descubrir un principio unificador dentro de la misma nación que fuese igualmente válido para la política interior.” (ARENDT, 1992, p. 78) La interiorización del enemigo externo en el pueblo-sujeto se logra por medio de la localización del enemigo en cada ciudadano bajo la forma de su voluntad particular que es a la vez identificada a su interés egoísta. Este enemigo interno particular se generaliza a enemigo del pueblo concentrando las voluntades individuales en la voluntad de todos, oponiendo los intereses particulares y egoístas de cada uno a los intereses generales de la Nación. Oponiendo no ya el nacional al extranjero sino el hombre al ciudadano.

La voluntad general presupone la sospecha y el combate de la voluntad individual " $" \mathrm{El}$ acuerdo entre dos intereses particulares se constituye por oposición a un tercero. " Hubiera podido añadir que la concordancia de todos los intereses se realiza en oposición al interés de cada uno. Si no hubiese intereses diferentes, apenas notaríamos la presencia del interés común, que jamás hallaría obstáculos; todo funcionaría por sí mismo y la política dejaría de ser un arte" (ROUSSEAU 1993, II, 3). Batalla del corazón que cada individuo está condenado a perder de una forma u otra: sometiendo su propia voluntad a la general, mecanismo por medio de la cual el hombre accede a ciudadano y gana su lugar en el cuerpo político soberano; o manteniendo su voluntad individual y por lo tanto alienándose de todos.

La única forma en que el individuo podría mantenerse ligado al cuerpo político es, en los términos de Rousseau, estar en constante rebelión contra sí mismo y contra su propio interés. Aquí debemos destacar que 


\section{8}

no se trata de suprimir u olvidar la existencia de los intereses particulares sino justamente de recordarla y dominarla. La noción del interés particular es absolutamente necesaria para percibir la voluntad general -que sólo se manifiesta en conflicto con el interés egoísta, generando a la vez compasión y apasionamiento- y es por eso que la estructura de la voluntad es, en palabras de Arendt, de mando y obediencia. Es necesario ser como un Dios para sí mismo, controlando y dominando los impulsos anti-sociales que todos albergamos en nuestra particularidad, es decir, en aquello que nos distingue de los demás.

Ahora bien, como observa Arendt, si los padecimientos del "pueblo" habían logrado colocar a una unidad en el lugar de una multitud, si por medio de la necesidad, el pueblo hablaba como uno, a los hombre de la revolución francesa aún les faltaba que aquellos que no sufrían las mismas carencias, que las clases privilegiadas social y políticamente, pudieran conformar una unidad con el pueblo. Es aquí donde, según Arendt, las ideas de Rousseau juegan un papel crucial en las experiencias de los revolucionarios. "No ofrecía dudas, para Robespierre, que la única fuerza que podía y debía unir a las diferentes clases de la sociedad de una nación era la compasión de los que no sufrían por les malheureux, la compasión de las clases altas por el pueblo bajo." (ARENDT, 1992, p. 81) La compasión era para Rousseau, la muestra de que algo de la bondad natural del hombre había sobrevivido a la perversión de la sociedad y, por lo tanto, constituía la mayor "virtud" de quienes no padecían esas carencias. La pitié de los revolucionarios era el sentimiento por medio del cual se veían unidos al pueblo y podían en todo derecho actuar en su nombre. Pero si la compasión y la piedad entraron de la mano de Rousseau en el ámbito de la política y se convirtieron en la "virtud" declarada de los revolucionarios por intermedio de Robespierre, ésta nunca tuvo la posibilidad de mostrarse en el espacio público ni de actuar sobre las injusticias. La compasión no comparte con las virtudes políticas su capacidad de aparecer a la luz de lo público a través de las acciones humanas como ejecuciones de un arte. Pertenecientes al corazón humano, necesitan de la oscuridad de la intimidad fuera de la cual se pervierten y se vuelven meras apariencias detrás de las cuales siempre se espera descubrir las verdaderas motivaciones de los actores. No sólo la compasión y la piedad se destruyen en contacto con la luz del espacio público sino que también 
tienen efectos destructivos sobre este espacio. "La compasión es, desde el punto de vista político, irrelevante e intrascendente, debido a que anula la distancia, el espacio mundano interhumano donde están localizados los asuntos políticos." (ARENDT, 1992, p. 87) Destruye el espacio de interrelación entre los hombres ya que los acerca, por así decir, más de la cuenta, haciendo que sea imposible dirigirse a alguien o mantener un discurso sobre algo que inter-esse, que literalmente se encuentre entre los hombres. De esta forma, la compasión sólo puede "hablar" o "actuar" en nombre del pueblo que, como ya habíamos visto, es despojado de estas capacidades y del espacio donde estas acciones se desarrollan. Pero aún es excesivo pensar que acciones o discursos pueden sostenerse a partir de la compasión. Es la violencia la que, según Arendt, hace su aparición en su nombre "Par pitié, par amour, pour l'humanité, soyez inhumain", son palabras que, tomadas casi al azar, de una de las peticiones presentadas por una sección de la Comuna de París a la Convención nacional [...] constituyen el auténtico lenguaje de la piedad.»(ARENDT, 1992, p. 90) Ese lenguaje que se abre frente al "abyecto espectáculo de la miseria" no tiene por objeto convencer o persuadir sino traducir las pasiones y sentimientos del pueblo en los dictámenes de la voluntad.

El pueblo, referente de la voluntad general, fuente de la ley, queda constituido como el Absoluto por medio del cual se justifica el poder ilimitado de la Nación. Nada puede sujetar a la voluntad general, que queda siempre liberada de sus límites y por lo tanto puede cambiar de dirección cuando quiera. Al respecto Arendt cita la sentencia de Rousseau: "es absurdo para la voluntad comprometerse a sí misma para el futuro" (Rousseau, J.-J., I, 7, citado en ARENDT, 1996, p. 176). Esto según Arendt, se traduce tanto en el fenómeno de que la voluntad del pueblo sea cambiante "como la de una persona" como en el hecho de que sea tan fácilmente apropiable. En efecto, para que la voluntad general pueda expresarse es necesario que el pueblo calle. Según Arendt "Rousseau [...] sostuvo que en un Estado ideal «los ciudadanos no tienen comunicación los unos con los otros»; que, para evitar que se organicen facciones "cada ciudadano debe pensar sólo sus propios pensamientos»." (ROUSSEAU, J.-J., II, 3, citado en ARENDT, 1996, p. 176) A los dictámenes de la voluntad general sólo pueden aparecer a un legislador que como una inteligencia superior que "viese todas las pasiones de los 
hombres sin experimentar ninguna; que no tuviese ninguna relación con nuestra naturaleza, conociéndola a fondo.” (ROUSSEAU, J.-J. 1993, II, 7)

Para redactar las leyes serían preciso dioses, hombres extraordinarios al Estado que, como los arquitectos de las polis griegas construyan las murallas que defiendan la ciudad y el edificio legislativo que preserve la res publica. Que logre cambiar la naturaleza humana "transformar a cada individuo que por sí mismo es un todo prefecto y solitario, en una parte de un todo más grande, del que recibe, en cierto modo, este individuo su vida y su ser" (ROUSSEAU, J.-J. 1993, II, 7). El problema, reconoce Rousseau, es que nadie autoriza a este legislador. Las leyes que él pueda redactar deben ser declaradas por los hombres, la vigencia de la ley y su fuerza coactiva depende de la reciprocidad de las leyes. "El pueblo mismo no puede, aunque quisiera, despojarse de ese derecho incomunicable: porque, según el pacto fundamental, sólo la voluntad general obliga a los particulares y jamás se puede asegurar que una voluntad particular está conforme con la voluntad general sino después de haberla sometido a los sufragios libres del pueblo." (ROUSSEAU, J.-J. 1993, II, 7) El problema entonces, se desdobla. El gran intérprete de la voluntad general no cuenta con autoridad alguna para hacer frente a una "empresa que esta por encima de la fuerza humana y para ejecutarla [cuenta con] una autoridad que no es nada" (ROUSSEAU, J.-J. 1993, II, 7). Toda su "autoridad" reside en su inteligencia y sagacidad para comunicar al pueblo lo que su propia voluntad le dicta, pero para que estas leyes tengan vigencias, es el pueblo quien debe refrendarlas. Rousseau concluye en que el legislador, si quiere que su empresa no caiga en desgracia, debe proclamarse intérprete de la voluntad divina. "No pudiendo emplear el legislador ni la fuerza ni el razonamiento, tiene por necesidad que recurrir a una autoridad de otro orden, que pueda arrastrar sin violencia y persuadir sin convencer [...] Esta razón sublime que se eleva por encima del alcance de los hombres vulgares es la que induce al legislador a atribuir las decisiones a los inmortales, para arrastrar por la autoridad divina, a aquellos a quienes no podría estremecer la prudencia humana." (ROUSSEAU, J.-J. 1993, II, 7)

\section{Conclusión: Soberanía versus liberdad}


La moderna teoría de la soberanía, de la cual "Jean-Jacques Rousseau siguió siendo el representante más sólido" (ARENDT, 1996, p. 176) se sostiene sobre este doble movimiento de inclusión y exclusión por medio del cual el hombre es fuente del poder y el Derecho, pero sólo al precio de olvidar el origen humano de las leyes y del Estado. Por medio de este subterfugio (HILB, 1990, p. 6) se introduce el principio absoluto en los asuntos humanos de los cuales nunca puede derivar la libertad. Al pretender regular la naturaleza humana y no las relaciones entre los hombres, las leyes no son determinadas por éstos sino que, a la inversa, los hombres son determinados por las leyes.

A través de la interpretación de la Revolución Francesa y de la crítica de las ideas rousseaunianas que allí encarnaron, Arendt sustenta su idea de la necesidad de separar radicalmente la soberanía de la libertad. "Cuando los hombres quieren ser soberanos, como individuos o como grupos organizados, deben rendirse a la opresión de la voluntad, ya sea la individual con la que cada uno se obliga a sí mismo, o la "voluntad general» de un grupo organizado. Si los hombres quieren ser libres, deben renunciar precisamente a la soberanía.” (ARENDT, 1996, p. 177)

Para acceder a una imagen de la libertad política moderna habrá que renunciar a la idea del pueblo soberano, unificado bajo la figura de un cuerpo colectivo poseedor de una voluntad general y reencontrar, señala Arendt, la imagen de un pueblo múltiple que es libre porque no es soberano. Si el pueblo de Rousseau reducía su soberanía al con-sentir con las leyes redactadas por el legislador, el pueblo que Arendt rescata de la Revolución americana, es aquel que actúa a través de con-prometerse con los demás en pactos y alianzas. Como sostiene E. Tassin, "Este pueblo no quiere. Este pueblo delibera y actúa.” (TASSIN, 2007, 314) De esta forma, la idea de poder político no se refleja en la falta de obstáculo a una voluntad sin límites ni divisiones, sino en la capacidad de acción concertada y por lo tanto limitada por las presencia de los otros.

${ }^{1}$ Aquí nos centraremos en la crítica que H. Arendt dedica al pensamiento e interpretación de J-J. Rousseau en las páginas de su obra Sobre la Revolución y en su artículo ¿Qué es la libertad?. Es notable que estas reflexiones estén basadas principalmente en las ideas del Contrato 


\section{2}

Social y, aunque también analiza fragmentos del Discurso sobre el origen y los fundamentos de la desigualdad de los hombres, pasan por alto otras obras de Rousseau. Es en este sentido que decimos que la crítica de Arendt no se centra en el pensamiento de Rousseau sino en las consecuencias que tuvo su obra, y El Contrato Social fue sin dudas una de las obras que más marcaron el pensamiento y la acción política en la modernidad.

2 "La Revolución había alcanzado su punto crítico cuando los jacobinos, bajo la dirección de Robespierre, se apoderaron del poder, no porque fuesen más radicales, sino debido a que no estaban interesados, como los girondinos, por las formas de gobierno, ya que creían más en el pueblo que en la república y «confiaban mucho más en la bondad natural de una clase» que en las instituciones y constituciones: «Bajo la nueva Constitución -repetía Robespierre- las leyes deben ser promulgadas 'en nombre del pueblo francés' y no en el de la 'República Francesa'.»” ARENDT 1992, Sobre la revolución, Buenos Aires, Alianza Editorial, p. 76-77.

\section{Referências bibliográficas}

ARENDT 1992, Sobre La Revolución, Buenos Aires, Ed. Alianza.

1996, ¿Qué es la libertad? in Entre El Pasado Y El Futuro.

Ocho Ejercicios Sobre La Reflexión Política., Barcelona, Ed. Península.

2007, ¿Qué es la política?, 1?Ed. 1? reimpresión, Buenos

Aires, Paidós.

Canovan, M. 1983, Arendt, Rousseau, and Human Plurality in Politics, The Jounal of Politics, Vol. 45, N?2, pp. 286-302, Mayo 1983, Cambridge University Press.

De Man, P. 1989, Allégories de la lecture. Le langage figuré chez Rousseau, Nietzsche, Rilke et Proust, Paris, Galilée.

Enegrén, A. 1994, Revolución y Fundación, In El resplandor de lo público. En torno a Hannah Arendt, Hilb, C. (comp.), Buenos Aires, Nueva Sociedad.

Hilb, C. 1990, Promesa y política. Promesas traicionadas y transición democrática, Buenos Aires, Conicet. 1992, Intra-muros.Y a-t-il un monde sans promesse? Les cahiers de philosophie, n? 15/16.

Honig, B. 2006, Declaration of independence. Arendt and Derrida on 
the problem of founding a republic, In Classical assessments of leading political philosophers, Garrath, W. (ed), Londres, Routhledge.

Rousseau, J.-J. 1993, El Contrato Social, Barcelona, Ed. Altaya.

TASSIN,. 2007, Le peuple ne veut pas. In Hannah Arendt: Crisis De L'état-Nation. Pensées Alternatives., Anne Kupiec, Martine Leibovici, Géraldine Muhlman Etienne Tassin organizadores, Paris: Sens\&Tonka, 2007. 